\title{
Development of newly designed combined grinding equipment
}

\author{
L. M. Gan, H. L. Gan \& A. B. Rosli \\ Faculty of Mechanical Engineering, Universiti Malaysia Pahang, \\ Malaysia
}

\begin{abstract}
As acknowledged, biomass has been identified as the most promising alternative for replacing fossil fuel due to its sustainability. Grinding is known to be essential to reduce the size of biomass samples in order to produce products with enhanced strength and durability. Some of the commonly used devices include a hammer mill and a knife mill. Bearing in mind the importance of such apparatus, a piece of grinding equipment has been designed and fabricated. However, the first prototype of the grinding facility resulted in irregular shape and sizes of the specimens, and consequently reduced the quality of the ground products obtained. This phenomenon therefore prompted the necessity to design a new facility. This paper thus discusses the development of the improved version of the grinding facility from the existing one through the incorporation of better functions. The improved version of the facility utilised powerful cutting blades with both shearing and hammering functions, which produced more uniformed and fine particles. A specially designed specimen collector was also added to the facility to reduce dust contamination. The assembled facility was utilised to be tested on biomass resources such as Jatropha fruits and oil palm stems. Through the manipulation of control parameters like cutting speed and operating time, the fineness of the particles could be varied, as appropriate.
\end{abstract}

Keywords: combined grinding, grinding, biomass, shearing, hammering.

\section{Introduction}

As found in studies, fossil fuels are currently facing depletion and as Gross et al. [1] has predicted, the resources will only last till the year 2030. This particular situation has induced an extensive search for alternative energies to counter the problem. Biomass has thus been identified as one of the most 
promising ones due to its environmental-friendliness and sustainability. On top of that, recent research have also proven the necessity of size reduction of the samples in order to preserve a good quality of the final products.

Grinding process refers to the process of reducing particle size through a various of methods, for instance cutting and hammering. Grinding is essential as finer particles would result in a better outcome of the final briquetted product, in terms of strength and durability. The application of pre-treatment for instance size reduction is critical to aid in increasing the surface area of the material prior to densification and consequently enhancing the inter-particle bonding [2]. In simpler words, the intention of grinding or commonly referred as size reduction is to transform the material into a form that optimizes handling, storage, transportation and finally conversion [3]. Grinding is usually achieved through the utilisation of different milling machines, either knife mill, hammer mill or disk mill, depending on the subsequent processes involved and the desired form of final products. As emphasised by Womac et al. [4], a degree of reduction in particle size would normally be required for all biomass processing and use.

The prototype grinding machine was relatively a simple facility consisting only of the hammering blades equipped with motor to cut the raw oil palm into finer particles. The main problem of the facility was the undesired quality of ground products formed in terms of particle fineness and shapes besides long period of time needed to cut the raw materials into a desired fineness. This phenomenon took place since only the hammering blades were utilised instead of proper ones, which meant the materials were hammered into finer particles without any shearing function. At the same time, the ground specimen resulted in dust contamination due to lack of proper storing compartment.

Thus, the studies for improvement meant to overcome the issues covered the utilisation of appropriate grinding blades having both the shearing and hammering functions and a specially designed specimen collector for ground specimens. The specimen collector would thus aid in minimising the dust contamination problem. Figure 1 depicts the first prototype grinding facility and its subsequent improved version.

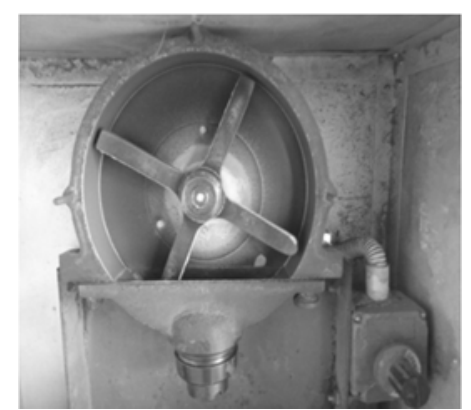

The grinding machine prototype

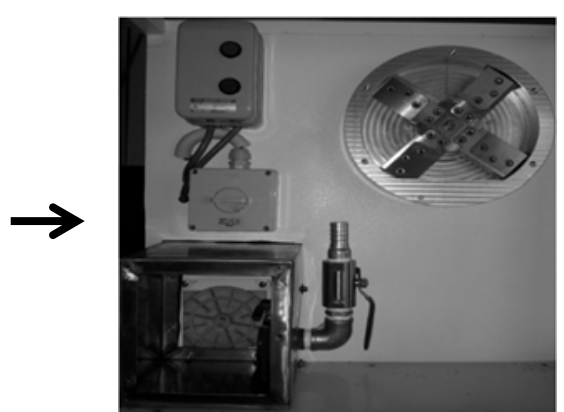

The improved version of the prototype

Figure 1: The first prototype grinding facility, followed by its improved version, especially in terms of the blade design after realising the shortcomings of the former. 


\section{Methodology}

The design of the grinding facility was performed using 3D modelling tool. The design stage was divided into a few sections, including the feeding section, grinding blade, grinding housing with bearings and others. Each section was then displayed in exploded view for a better view. Figure 2 portrays the exploded view of the facility emphasising a few sections in particular, namely the grinding blades with holders, feeding section as well as grinding housing with bearings. Each section was consisted of numerous components, leading to the complete facility eventually.

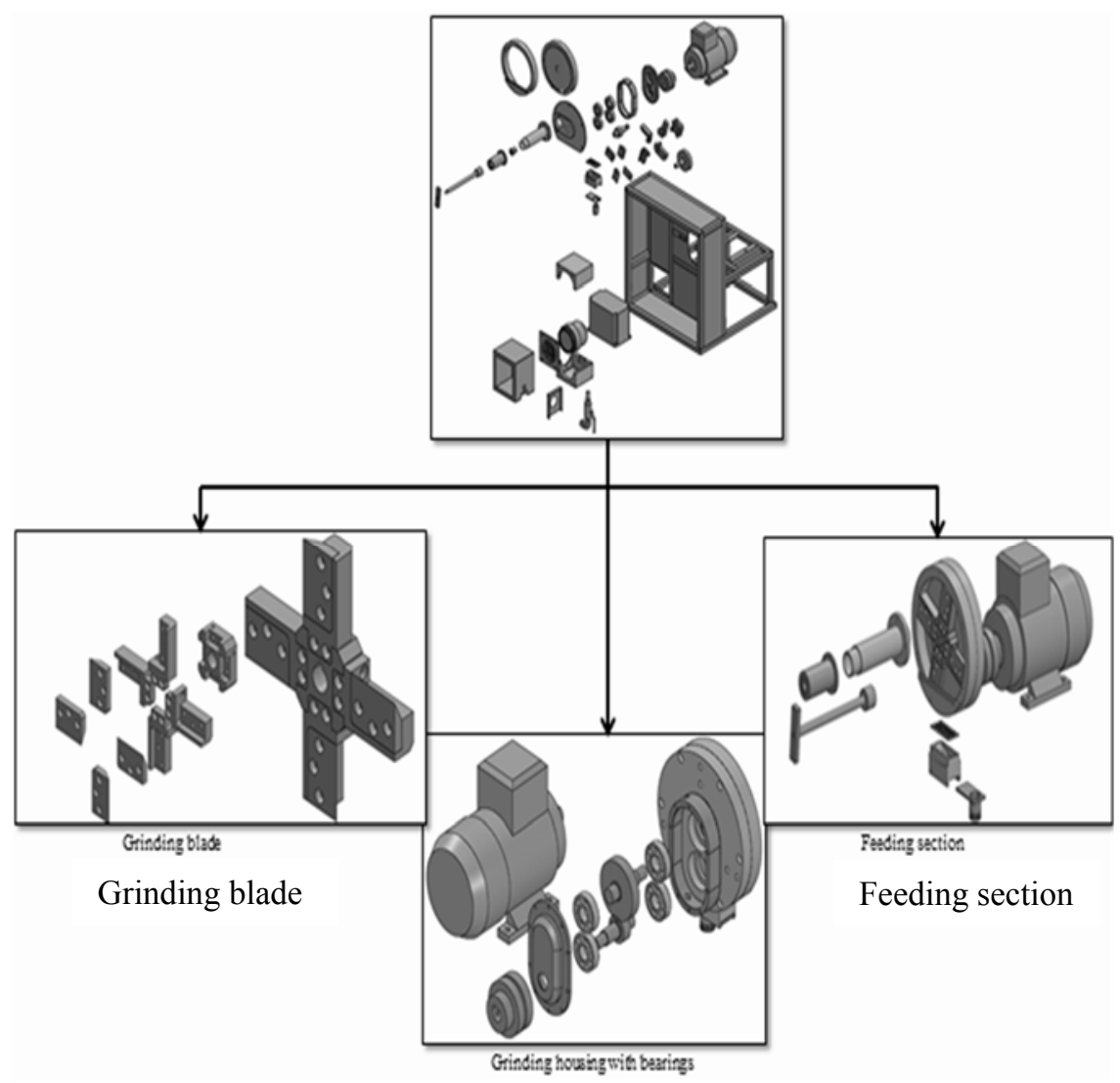

Grinding housing with bearings

Figure 2: Exploded view of the grinding facility, with emphasis to the grinding blade, grinding housing with bearings and feeding section.

A typical grinding blade would normally be equipped with the cutting or shearing function. However, those blades could only result in coarser particles and irregular size. As a result, the hammering function was then introduced as to aid 
in the production of finer and more uniform particles. The outcomes of the ground specimens can be found in the Results and Discussion section.

Next, the working principle of the facility is shown in Figure 3. First of all, the samples would be fed into the sampling pipe by lifting the push piston to the rest position. The piston would then be moved to the close position and slowly moved inwards till its end. That would drive the samples to be fed to the cutting blades and subsequently cut into finer particles. Finally, the grinded particles would be driven to the outlet and collected until it got transferred for the next procedure.
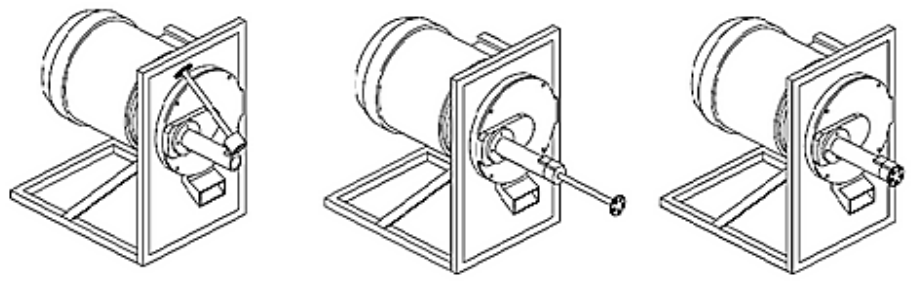

Figure 3: Working principle of the grinding machine.

Besides the mechanical work, electrical work was also necessary in order to run the machine. The main components in the electrical system would be the $5 \mathrm{hp}$ motor and the inverter used to control the speed of the machine. As shown in Figure 4, the main switch was connected to the inverter and the wall plug. The L1, L2 and L3 terminals were connected to brown, black and blue terminals of the main switch, respectively. Then the terminals from the main switch were connected to the L1 (brown), L2 (black) as well as L3 (blue) terminals of the socket. The inverter was set using the function code of b00 indicating external control, as described in Table 1.

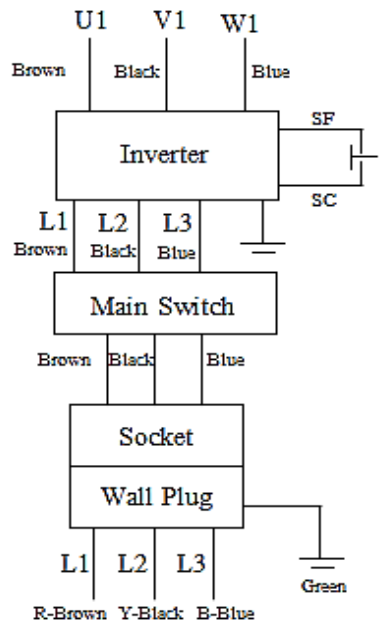

Figure 4: Schematic wiring diagram for the machine. 
Table 1: Inverter setting.

\begin{tabular}{|l|l|l|l|}
\hline Function & Function code & Description & Parameter range and description \\
\hline $\begin{array}{l}\text { Running } \\
\text { command } \\
\text { source }\end{array}$ & b00 & Set the source of & 4: External via control terminals \\
& & $\begin{array}{l}\text { commands for } \\
\text { running (option 1 \& } \\
\text { 3 frequency } \\
\text { command source) }\end{array}$ & $\begin{array}{l}\text { internal/external sources of } \\
\text { frequency command, while } \\
\text { STOP key is activated) }\end{array}$ \\
\hline
\end{tabular}

\section{Results and discussion}

The facility consisted of 22 main components, including specimen duct and outlet, specimen piston and its guide, specimen filter plate, grinding blade with holder, main housing, as well as gearbox housings. Most of the components were manufactured using light-weight, high quality stainless steel possessing good strength. The individual components and the assembled facility are clearly depicted in Figure 5. The Bill of Materials (BOM) is shown in Table 2.

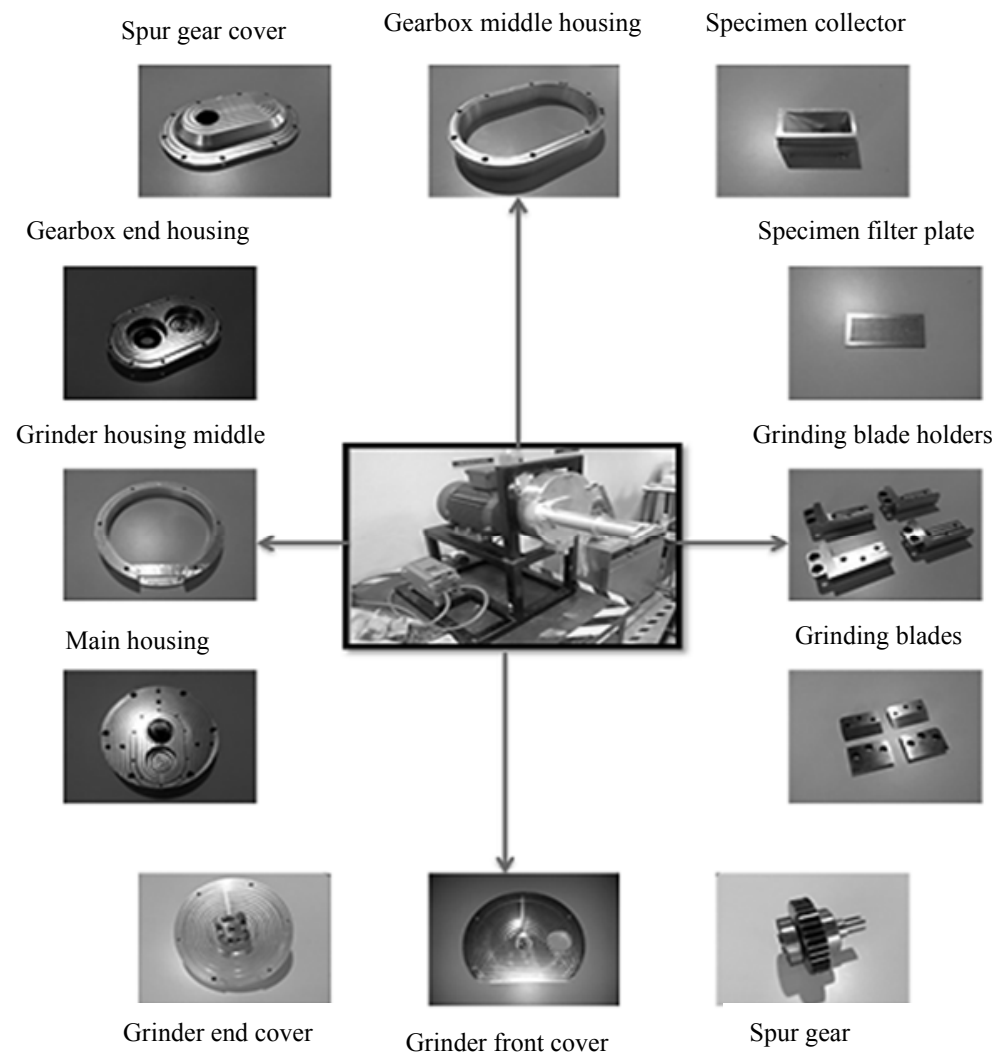

Figure 5: Exploded view of the fabricated facility. 
Table 2: BOM table.

\begin{tabular}{|l|c|l|}
\hline Component & Quantity & Material \\
\hline Main housing & 1 & Stainless steel \\
\hline Grinder housing middle & 1 & Stainless steel \\
\hline Grinder end cover & 1 & Stainless steel \\
\hline Grinder front cover & 1 & Stainless steel \\
\hline Grinding blade holder & 4 & Stainless steel \\
\hline Grinding blade & 4 & Stainless steel \\
\hline Gearbox middle housing & 1 & Stainless steel \\
\hline Gearbox end housing & 1 & Stainless steel \\
\hline Specimen collector & 1 & Stainless steel \\
\hline Specimen collector filter & 2 & Stainless steel \\
\hline Spur gear & 2 & Stainless steel \\
\hline Spur gear cover & 1 & Stainless steel \\
\hline Bearing & 4 & Standard bearing steel \\
\hline Push piston & 1 & Carbon steel with acrylic \\
\hline Feeding pipe & 1 & Carbon steel \\
\hline Structural frame & 1 & Hollow square steel \\
\hline Coupling & 1 & Standard material \\
\hline Connecting bar & 1 & Stainless steel \\
\hline Fasteners & - & Stainless steel cap or hex \\
\hline Inverter & 1 & - \\
\hline 5hp motor & 1 & - \\
\hline
\end{tabular}

Figure 6 gives a clearer picture on the shearing and hammering functions of the grinding blades as the functions would be the main concern of the improvement.

As portrayed, the new design of the blades was comprised of the functions of shearing and hammering. That meant a sample fed into the sampling pipe would first be sheared to a certain particle size before got hammered into finer particles. With that, the subsequent quality of the products could be preserved. The cutting frequency ranged from $10 \mathrm{~Hz}$ to $50 \mathrm{~Hz}$, through the control on the inverter.

However, it shall be remembered that skills would be required during the installation as to prevent damages to any of the components, particularly the bearings and blades. Caution was given at all times while inserting the bearings and grinding blades into the respective locations as they were indeed very susceptible to damage once dropped or knocked accidentally. At the same time, the bearings would need to be lubricated with gear oil as to prevent them from being overheated, expanded and got damaged. The oil would be added through the oil seal whenever necessary.

The assembled facility was then tested with various types of samples, inclusive of clumped soil, Jatropha fruits and oil palm stems. Figure 7 shows the grinding outcome for the clumped soil while Figure 8 depicts the dried Jatropha fruits and oil palm stems before and after grinding at medium speed. Finally, Figure 9 gives a clear picture on the varying fineness of the particles formed when control parameters are manipulated. 


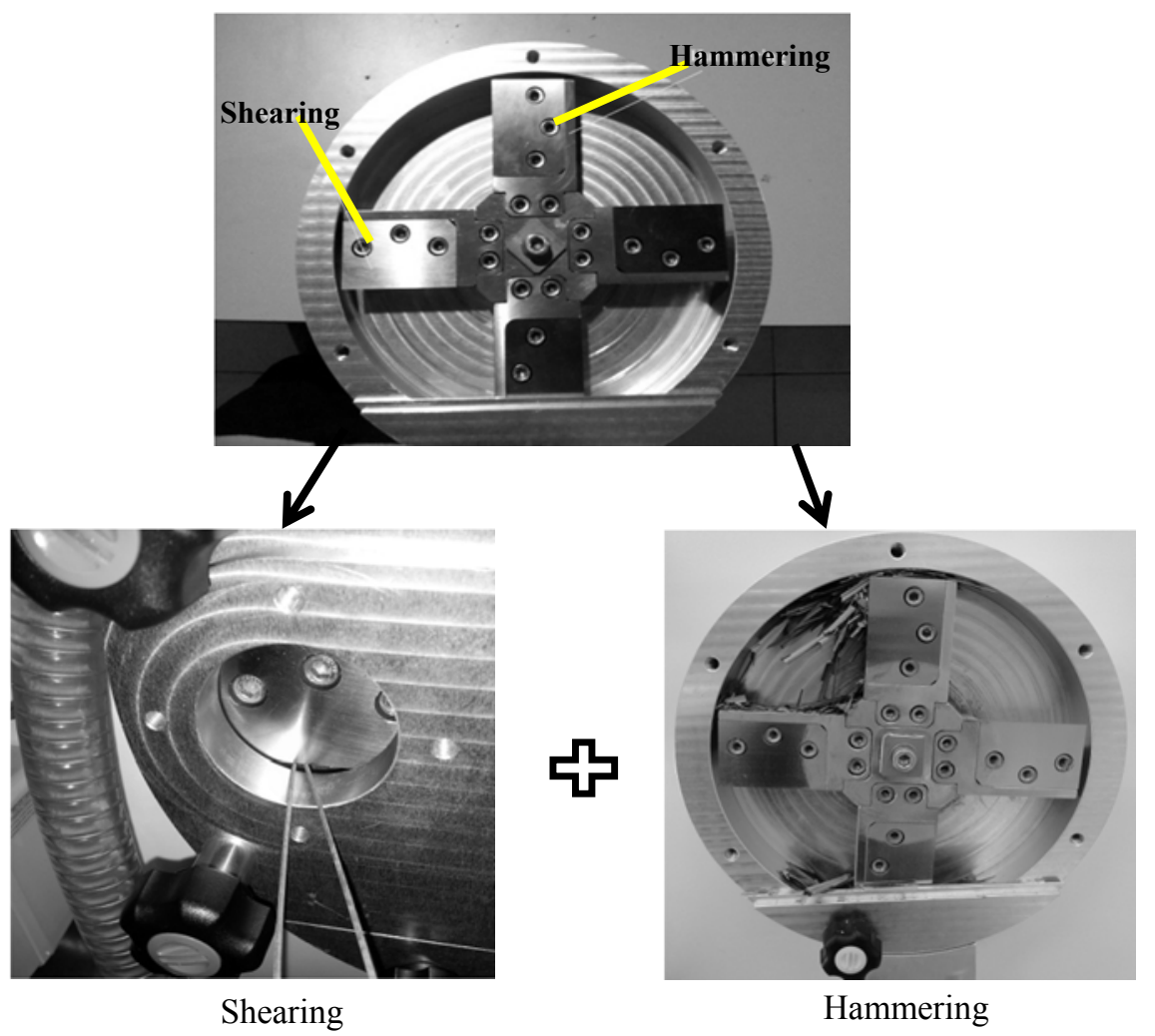

Figure 6: Detailed view of the blades with shearing and hammering functions.

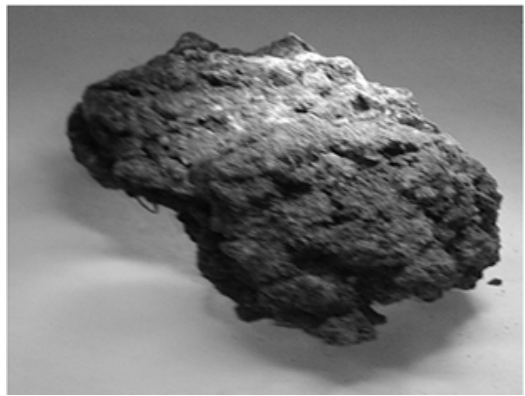

(a) Original sample

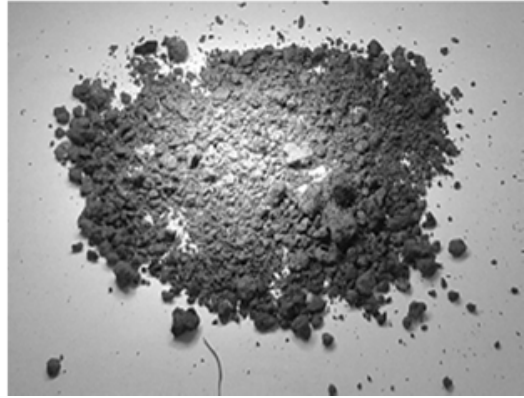

(b) Ground sample

Figure 7: Outcome (a) before and (b) after the grinding process. 


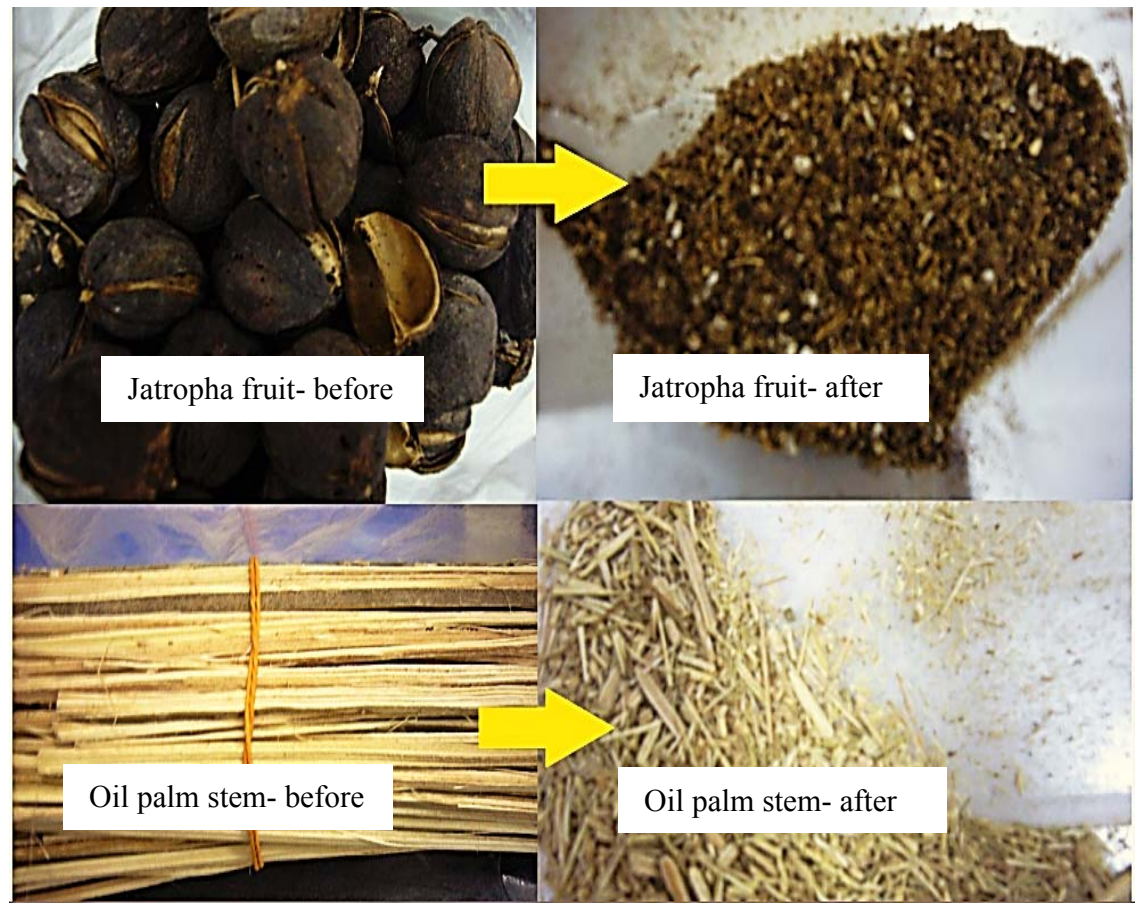

Figure 8: Jatropha fruits (upper) and oil palm stems (bottom), before and after undergoing grinding at medium speed of $25-30 \mathrm{~Hz}$.

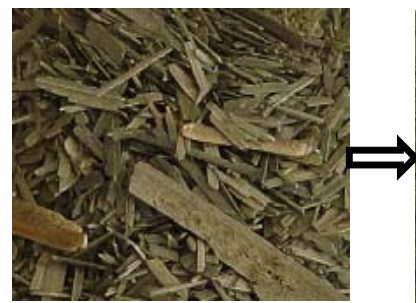

(a) Coarse

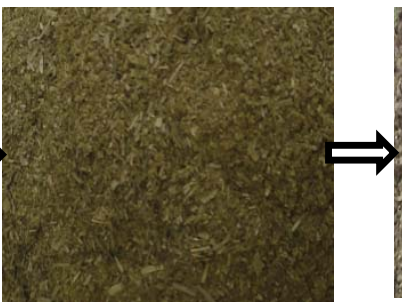

(b) Medium

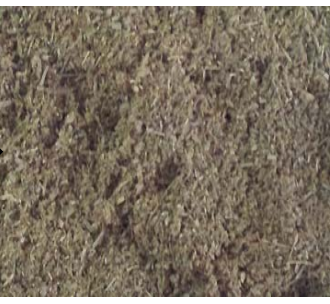

(c) Fine

Figure 9: Figure 9: Varying size of particles (from (a) coarse to (c) fine) resulting from the grinding process.

As seen in the figures, the initial large and particularly hard samples were ground to fine and scattered powdery form, which could be used for subsequent densification process. As acknowledged, the size reduction process could not be effective enough if a manual grinding was to be opted. This extent of fineness could only be achieved when the cutting blades were properly designed. The outcomes therefore proved the effectiveness of the newly-designed blades. 


\section{Further study}

The facility should be tested with more samples as to observe its effectiveness on different types of materials. The particle size could be identified by using delicate equipment for example Scanning Electron Microscope (SEM) or FESEM (Field Emission Scanning Electron Microscope).

\section{Acknowledgement}

The highest appreciation to my supervisor Dr Gan Leong Ming for his continuous guidance.

\section{References}

[1] Gross R., Leach M. and Bauen A. 2003. Progress in Renewable Energy Environment International 29, 105-122.

[2] Lape T. and Greg S. 2011. Grinding Performance and Physical Properties of Non-treated and Steam Exploded Barley, Canola, Oat and Wheat Straw. Biomass and Bioenergy 35, 549-561.

[3] Miao Z., Tony E. G. and Ting K. C. 2014. Size Reduction and Densification of Lignocellulosic Biomass Feedstock for Biopower, Bioproducts, and Liquid Biofuel Production. Encyclopedia of Agricultural, Food, and Biological Engineering, Second Edition. Taylor \& Francis.

[4] Womac A. R., Igathinathane C., Bitra P., Miu P., Yang T., Sokhansanj S. and Narayan S. 2007. Biomass Pre-Processing Size Reduction with Instrumented Mills. American Society of Agricultural and Biological Engineers (ASABE) Annual International Meeting. 